\title{
Compared to women without obesity, women with obesity have reduced odds of breastfeeding initiation and breastfeeding at hospital discharge
}

\section{Abstract}

Women with obesity breastfeed for shorter durations than women of normal-weight body mass index (BMI). There is also evidence to suggest that women with obesity are less likely to initiate breastfeeding. This is important because breastfeeding is associated with a small, but significant, reduced risk of obesity among offspring; which may be particularly important for the offspring of mothers with obesity as these infants are at an increased risk of obesity. Using data prospectively collected during 2015 by medical staff in a large maternity hospital, we aimed to explore the association between maternal obesity status and early breastfeeding outcomes: breastfeeding initiation and exclusive breastfeeding at hospital discharge (approx. day 2 of life). Data were obtained from electronic records for all births in The National Maternity Hospital, Dublin in 2015. We included data from mothers who delivered a healthy, full-term, singleton infant $(\mathrm{n}=7449)$. At the booking visit (approx. 12 weeks' gestation), maternal height and weight were objectively measured by nursing staff and subsequently used to calculate BMI; other demographic data were recorded at this time. At delivery, epidural use, mode of delivery, and infant sex and weight were recorded. At discharge, mode of feeding was recorded. In SPSS, we explored unadjusted and adjusted associations between obesity status (BMI $<30 \mathrm{~kg} / \mathrm{m}^{2}[\mathrm{n}=964] v s$. BMI $\left.\geq 30 \mathrm{~kg} / \mathrm{m}^{2}[\mathrm{n}=6485]\right)$ and breastfeeding outcomes (dichotomous: yes/no) using Chi-squared analysis and multivariate logistic regression, respectively. In unadjusted analyses, there was a significant association between obesity status and breastfeeding initiation; $76 \%$ of women without obesity initiated breastfeeding compared with $59 \%$ of women with obesity $(P<0.001)$. There was also a significant association between obesity status and exclusive breastfeeding; $69 \%$ of women without obesity were exclusively breastfeeding at discharge compared with $37 \%$ of women with obesity $(P<0.001)$. In logistic regression analyses adjusted for maternal age, ethnicity, marital status, smoking status, parity, epidural use and delivery mode, the odds of women with obesity initiating breastfeeding were $51 \%$ lower than women without obesity (odds ratio [OR] $0.49,95 \%$ confidence interval [CI] $0.42-0.57 ; P<0.001$ ). Similarly, in adjusted analyses, the odds of women with obesity exclusively breastfeeding at hospital discharge were $54 \%$ lower than women without obesity (OR $0.44,95 \%$ CI $0.38-0.51$; $P<0.001)$. Research is needed to understand whether the negative association between obesity and breastfeeding is biological or cultural in origin, or both. Interventions to promote and support breastfeeding among women with obesity may improve breastfeeding outcomes.

\section{Conflict of Interest}

There is no conflict of interest. 